\title{
A PRACTICAL GREEN SYNTHESIS OF THIAZINE DERIVATIVES USING PHASE TRANSFER CATALYST
}

\author{
Varsha V. Bankar and Raksha P. Dhankar* \\ Centre for Higher Learning and Research, Department of Chemistry, \\ Sardar Patel Mahavidyalaya, Chandrapur, 442401, Maharashtra, India \\ *E-mail: rakshadhankar@rediffmail.com
}

\begin{abstract}
An operationally simple and environmental benign approach for the synthesis of thiazines under catalysis of tetrabutylammonium bromide (TBAB) as phase transfer catalyst (PTC) in the dichloromethane-water biphasic solvent system has been explored . This reaction has generated renewed interest in preparing thiazines.. Mild reaction conditions, enhanced rates, improved efficiency of reaction with maximum atom economy, use of an inexpensive catalyst, green solvent system and reduced reaction time are the salient features of our process. Our protocol extends the benefits to synthesis of 5-(2-amino-6-(3,4-substituted phenyl)-5,6-dihydro-4H-1,3-thiazine-4yl)-6-methyl-4-(substituted tolyl)-3,4-dihydropyrimidine-2(1H)-one derivatives in excellent yield. The structures of all the synthesized compounds have been confirmed by elemental analysis, FT-IR, ${ }^{1} \mathrm{HNMR},{ }^{13} \mathrm{C}$ NMR and mass spectral data.
\end{abstract}

Keywords: Thiazines, Phase transfer catalyst (TBAB), Green catalysis

(C) RASĀYAN. All rights reserved

\section{INTRODUCTION}

Phase-transfer-catalyzed reactions are environmentally-friendly processes due to their simplicity, mild conditions and low-cost performance ${ }^{1}$. The methodology of phase-transfer catalysis involves an organic soluble reactant and an aqueous-soluble anionic reactant; and these water-soluble anions are then brought together by a catalyst, which transports the anion into the organic phase where the reaction takes place. Quaternary ammonium salts with their unique capability to dissolve in both aqueous and organic liquids are the catalysts of choice for most phase-transfer applications. The benefits of PTC lie in the elimination of organic solvents and dangerous or expensive bases, together with the simplicity of the procedure, and its high yields and the purity of the products ${ }^{2}$.

Heterocyclic chemistry research encompasses almost half of the organic chemistry research throughout the whole world ${ }^{3,4}$. Heterocyclic thiazine derivatives with nitrogen and sulphur as heteroatoms are important because they are biological constituents of many biomolecules and drugs ${ }^{5}$. Some derivatives of thiazine are cannabinoid receptor agonists, also they can act as an antihypotensive, antitubercular and antibacterial agents Moreover, thiazine derivatives can be used for gastrointestinal disorders or diabetes prevention $^{6-11}$

We herein report a new method for the synthesis of 5-(2-amino-6-(3,4-substituted phenyl)-5,6-dihydro4H-1,3-thiazine-4-yl)-6-methyl-4-(substituted tolyl)-3,4-dihydropyrimidine-2(1H)-one under TBAB as phase transfer catalyst in $\mathrm{CH}_{2} \mathrm{Cl}_{2}$-water solvent system. TBAB showed optimal conversion and highest reaction efficiencies as compared to other phase transfer catalysts. Our designed benign reaction does not generate any toxic waste product.

\section{Materials and Methods}

\section{EXPERIMENTAL}

Substituted Aldehydes, thiourea,tetrabutyl ammonium bromide (TBAB), PEG 400, $\beta$-cyclodextrin, dichloromethane, ethanol, ethyl acetate and n-hexane were obtained from Qualigen India Ltd. Mumbai.

Rasayan J. Chem., 11(3), 1294-1299(2018)

http://dx.doi.org/10.31788/RJC.2018.1134012

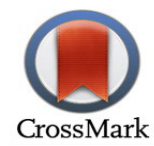


RASĀYAN J. Chem.

Vol. 11 | No. 3 |1294 - 1299 | July - September | 2018

\section{Apparatus}

Thin layer chromatography was performed on silica gel G. Melting points were determined by the open capillary method. IR spectra were recorded using a Shimadzu FT-IR spectrometer using KBr pellets. GC/MS analysis was carried out using GC model: Shimadzu gas chromatograph coupled with a QP5050 spectrometer at 1-1.5ev. Proton and NMR spectra were recorded on a Brucker AVII FT-NMR spectrometer operating at $400 \mathrm{MHz}$ for the entire sample.

\section{General Procedure for the Synthesis of 5-Cinnamoyl-6-methyl-4-phenyl -3,4-dihydropyrimidine 2(1H)-one (1a-j)}

The synthesis of chalcones was carried out via Claisen -Schmidt condensation. A mixture of 5-acetyl-6 methyl-4-phenyl-3,4 dihyropyrimidine $-2(1 \mathrm{H})$-one $(1 \mathrm{mmol}, 0.214 \mathrm{~g})$ and benzaldehyde $(1 \mathrm{mmol}, 0.106 \mathrm{~mL})$ was dissolved in $10 \mathrm{~mL}$ of ethanol in $250 \mathrm{~mL}$ round bottom flask equipped with magnetic stirrer. Then 20 $\mathrm{mL} \mathrm{NaOH}$ solution ( $8 \mathrm{~g}$ in $20 \mathrm{~mL} \mathrm{H}_{2} \mathrm{O}$ ) was added drop wise to the reaction mixture on vigorous stirring for 30 mins at room temperature and kept this reaction mixture overnight. The reaction mixture was neutralized by adding dil. $\mathrm{HCl}$ whereby the precipitation occurred. The product was filtered and recrystallized by ethanol.

General Procedure for Synthesis of 5-(2-amino-6-(3,4-dimethylphenyl)-5,6-dihydro-4H-1,3thiazine-4-yl)-6-methyl-4-(P-tolyl)-3,4-dihydropyrimidine-2(1H)-one.(2a-j)

5-cinnamoyl-6-methyl-4-phenyl-3,4-dihydropyrimidin- $2(1 \mathrm{H})$ one, $(0.01 \mathrm{mmol}, 3.30 \mathrm{~g})$ thiourea $(0.02 \mathrm{mmol}$ , $1.52 \mathrm{~g}$ ) were introduced into a $50 \mathrm{~mL}$ round bottom flask. To this dichloromethane and water in a ratio of 2:1 was added slowly and stirred for 5 minutes. TBAB (30 mole $\%, 0.096 \mathrm{~g})$ was added with constant stirring at $50^{\circ} \mathrm{C}$. The stirring was carried out for $1 / 2$ an hour. The extent of the reaction was monitored by TLC. After the completion of the reaction, the reaction mixture was worked up in ice cold water. The product that separated out was filtered. The filtrate was evaporated to remove water leaving TBAB behind. The same TBAB was utilized for a further run.<smiles></smiles>

$1 \mathbf{a}$

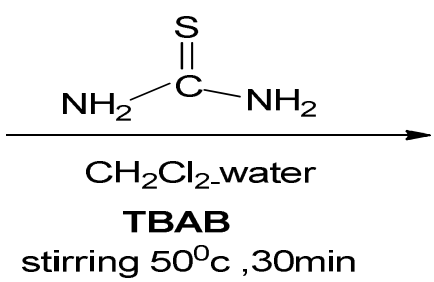

stirring $50^{\circ} \mathrm{C}, 30 \mathrm{~min}$<smiles>[R]c1ccc(C2NC(=O)NC(C)=C2C2CC(c3ccc([R2])c([R3])c3)SC(N)=N2)cc1</smiles>

$2 \mathbf{a}$

Scheme-1

$$
\mathrm{R}_{1}=\mathrm{H}, \mathrm{OH}, \mathrm{NO}_{2}, \mathrm{OCH}_{3} \mathrm{Cl} \quad \mathrm{R}_{2}=\mathrm{H}, \mathrm{NO}_{2}, \mathrm{OCH}_{3}, \mathrm{Cl} \mathrm{R}_{3}=\mathrm{H}
$$

Synthesis of 5-(2-amino-6-(3,4-dimethylphenyl)-5,6-dihydro-4H-1,3-thiazine-4-yl)-6-methyl-4-(Ptolyl)-3,4-dihydropyrimidine-2(1H)-one (2a)

Canary yellow; Yield $84 \%$; m.p. $=192^{\circ} \mathrm{C}\left[\mathrm{C}_{21} \mathrm{H}_{21} \mathrm{~N}_{4} \mathrm{OS}\right] \mathrm{IR}\left(\mathrm{KBr}, \lambda \max / \mathrm{cm}^{-1}\right) 3210,1680 \mathrm{~cm}^{-}$ ${ }^{1} \mathrm{HNMR}\left(400 \mathrm{MHz}, \mathrm{CDCl}_{3}\right) \delta(\mathrm{ppm})=\delta 2.28\left(\mathrm{~s}, 3 \mathrm{H}, \mathrm{CH}_{3}\right) ; 2.48-2.50\left(\mathrm{~d}, 2 \mathrm{H}, \mathrm{CH}_{2}\right)(\mathrm{J}=8 \mathrm{~Hz}) ; 3.1(\mathrm{t}, 1 \mathrm{H}, \mathrm{CH})$; 3.85(s, $\left.2 \mathrm{H}, \mathrm{NH}_{2}\right) ; 5.56(\mathrm{~s}, 1 \mathrm{H}, \mathrm{CH}) ; 7.1-7.6(\mathrm{~m}, 10 \mathrm{H}, \mathrm{Ar}-\mathrm{H}) ; 8.5(\mathrm{~s}, 1 \mathrm{H}, \mathrm{NH}) ; 8.7(\mathrm{~s}, 1 \mathrm{H}, \mathrm{NH})$. MS (70 eV): $m / z=377.14[\mathrm{M}+]$., found: 377.12; Anal. Calcd. for $\mathrm{C}_{21} \mathrm{H}_{21} \mathrm{~N}_{4} \mathrm{OS}$ 377.14: C, 66.82; $\mathrm{H}, 5.61$; N, 14.84; O,4.24; S,8.49. Found: C, 66.70; H, 5.40; N, 13.45; O, 3.43. S, 7.39. ${ }^{13} \mathrm{C}$ NMR $\left(\mathrm{CDCl}_{3}\right): \delta=15.4,36.6$, $42.3,58.8,115.8,123.5,126.0,126.7,126.8,126.9,139.5,141.9,150.2,159.3$.

Synthesis of 5-(2-amino-6-(3 methyl-4-nitrophenyl)-5,6-dihydro-4H-1,3-thiazine-4-yl)-6-methyl-4(P-tolyl)-3,4-dihydropyrimidine-2(1H)-one(2b)

Laser yellow; Yield $75 \% \mathrm{mp}=210^{\circ} \mathrm{C}\left[\mathrm{C}_{21} \mathrm{H}_{20} \mathrm{~N}_{5} \mathrm{O}_{3} \mathrm{~S}\right] \mathrm{IR}\left(\mathrm{KBr}, \lambda \max / \mathrm{cm}^{-1}\right) 3215,1670 \mathrm{~cm}^{-1}{ }^{1} \mathrm{HNMR}$ $\left(400 \mathrm{MHz}, \mathrm{CDCl}_{3}\right) \delta(\mathrm{ppm})=\delta 2.28\left(\mathrm{~s}, 3 \mathrm{H}, \mathrm{CH}_{3}\right) ; 2.48-2.50\left(\mathrm{~d}, 2 \mathrm{H}, \mathrm{CH}_{2}\right)(\mathrm{J}=8 \mathrm{~Hz}) ; 3.1(\mathrm{~s}, 1 \mathrm{H}, \mathrm{CH}) ;$ 


\section{RASĀYAN J. Chem.}

Vol. 11 | No. 3 |1294 - 1299 | July - September | 2018

3.85(s,2H, $\left.\mathrm{NH}_{2}\right) ; 5.56(\mathrm{~s}, 1 \mathrm{H}, \mathrm{CH}) ; 7.2-7.5(\mathrm{~m}, 7 \mathrm{H}, \mathrm{Ar}-\mathrm{H}) ; 8.2\left(\mathrm{~s}, 2 \mathrm{H}, \mathrm{CH}_{2}\right) ; 8.5\left(\mathrm{~d}, 2 \mathrm{H}, \mathrm{NH}_{2}\right)$. MS (70 eV): $m / z=422.13[\mathrm{M}+]=100 \%$., found: 422.13; Anal. Calcd. for $\mathrm{C}_{21} \mathrm{H}_{20} \mathrm{~N}_{5} \mathrm{O}_{3} \mathrm{~S}$,422.13: C, 59.70; H, 4.77; N, 16.58; O,11.36; S,7.59. Found: C, 56.70; H, 3.92; N, 15.45; O, 11.26. S, 6.39. ${ }^{13} \mathrm{C}^{\mathrm{N} M R}\left(\mathrm{CDCl}_{3}\right): \delta=$ $15.4,36.6,42.3,58.8,115.8,123.5,126.7,126.8,126.9,141.9,145.6,150.2,159.3$.

Synthesis of 5-(2-amino-6-(4 methoxy-3-methylphenyl)-5,6-dihydro-4H-1,3-thiazine-4-yl)-6-methyl4-(P-tolyl)-3,4-dihydropyrimidine-2(1H)-one $(2 \mathrm{c})$

Goldenrod yellow; Yield 79\% $\mathrm{mp}=205^{\circ} \mathrm{C}\left[\mathrm{C}_{22} \mathrm{H}_{23} \mathrm{~N}_{4} \mathrm{O}_{2} \mathrm{~S}\right] \mathrm{IR}\left(\mathrm{KBr}, \lambda \max / \mathrm{cm}^{-1}\right) 3210,1690 \mathrm{~cm}^{-1}{ }^{1} \mathrm{HNMR}$ $\left(400 \mathrm{MHz}, \mathrm{CDCl}_{3}\right) \delta(\mathrm{ppm})=\delta 2.28\left(\mathrm{~s}, 3 \mathrm{H}, \mathrm{CH}_{3}\right) ; 2.48-2.50\left(\mathrm{~d}, 2 \mathrm{H}, \mathrm{CH}_{2}\right)(\mathrm{J}=8 \mathrm{~Hz}) ; 3.1(\mathrm{~s}, 1 \mathrm{H}, \mathrm{CH}) ; 3.85(\mathrm{~s}, 2 \mathrm{H}$, $\left.\mathrm{NH}_{2}\right) ; 5.56(\mathrm{~s}, 1 \mathrm{H}, \mathrm{CH}) ; 6.9-7.4(\mathrm{~m}, 10 \mathrm{H}, \mathrm{Ar}-\mathrm{H}) ; 8.5\left(\mathrm{~d}, 2 \mathrm{H}, \mathrm{NH}_{2}\right) \mathrm{MS}(70 \mathrm{eV}): \mathrm{m} / z=407.15[\mathrm{M}+]=100 \%$., found: 407.15; Anal. Calcd. for $\mathrm{C}_{22} \mathrm{H}_{23} \mathrm{~N}_{4} \mathrm{O}_{2} \mathrm{~S}$,407.15: C, 64.84; H, 5.69; N, 13.75; O,7.85; S,7.87. Found: $\mathrm{C}, 62.70 ; \mathrm{H}, 6.92 ; \mathrm{N}, 12.45 ; \mathrm{O}, 7.49 . \mathrm{S}, 7.39 .{ }^{13} \mathrm{C} \mathrm{NMR}\left(\mathrm{CDCl}_{3}\right): \delta=15.4,36.6,42.3,44.3,55.8,58.8$, $114.4,115.8,123.5,126.7,126.9,131.8,141.9,150.2,159.3,157.9$.

Synthesis of 5-(2-amino-6-(4 methoxy-3-methylphenyl)-5,6-dihydro-4H-1,3-thiazine-4-yl)-4-(4methoxyphenyl)6-methyl-3,4-dihydropyrimidine-2(1H)-one(2d)

Munsell yellow Yield $80 \% \mathrm{mp}=232^{\circ} \mathrm{C}\left[\mathrm{C}_{23} \mathrm{H}_{25} \mathrm{~N}_{4} \mathrm{O}_{3} \mathrm{~S}\right] \mathrm{IR}\left(\mathrm{KBr}, \lambda \max / \mathrm{cm}^{-1}\right) 3210,1675 \mathrm{~cm}^{-1}{ }^{1} \mathrm{HNMR}$ $\left(400 \mathrm{MHz}, \mathrm{CDCl}_{3}\right) \delta(\mathrm{ppm})=\delta 2.28\left(\mathrm{~s}_{3} \mathrm{H}-\mathrm{CH}_{3}\right) ; 2.48-2.50\left(\mathrm{~d}, 2 \mathrm{H}, \mathrm{CH}_{2}\right)(\mathrm{J}=8 \mathrm{~Hz}) ; 3.1(\mathrm{~s}, 1 \mathrm{H}, \mathrm{CH}) ; 3.85(\mathrm{~s}, 2 \mathrm{H}$, $\left.\mathrm{NH}_{2}\right) ; 5.56(\mathrm{~s}, 1 \mathrm{H}, \mathrm{CH}) ; 6.8-7.2(\mathrm{~m}, 8 \mathrm{H}, \mathrm{Ar}-\mathrm{H}) ; 8.5\left(\mathrm{~d}, 2 \mathrm{H}, \mathrm{NH}_{2}\right) ; \mathrm{MS}(70 \mathrm{eV}): \mathrm{m} / \mathrm{z}=437.16[\mathrm{M}+]=100 \%$., found: 437.16; Anal. Calcd. For $\mathrm{C}_{23} \mathrm{H}_{25} \mathrm{~N}_{4} \mathrm{O}_{3} \mathrm{~S}$, 435.25: C, 63.84; H, 5.76; N, 12.81; O, 10.97; S,7.33. Found: C, 62.70; H, 5.90; N, 11.45; O, 6.49.S, 5.39. ${ }^{13} \mathrm{C}$ NMR $\left(\mathrm{CDCl}_{3}\right): \delta=15.4,36.6,42.3,44.5,55.8$, $114.1,114.4,115.8,123.5,125.7,129.1,131.8,150.2,157.9,159.3$.

Synthesis of 5-(2-amino-6-(3,4 dimethylphenyl)-5,6-dihydro-4H-1,3-thiazine-4-yl)-4-(4chlorophenyl)-6-methyl-3,4-dihydropyrimidine-2(1H)-one (2e)

Jonquil yellow Yield $65 \% \mathrm{mp}=215^{0} \mathrm{C}\left[\mathrm{C}_{21} \mathrm{H}_{20} \mathrm{ClN}_{4} \mathrm{O}_{5} \mathrm{~S}\right] \mathrm{IR}\left(\mathrm{KBr}, \lambda \max / \mathrm{cm}^{-1}\right) 3217,1672 \mathrm{~cm}^{-1}{ }^{1} \mathrm{HNMR}$ $\left(400 \mathrm{MHz}, \mathrm{CDCl}_{3}\right) \delta(\mathrm{ppm})=\delta 2.28\left(\mathrm{~s}, 3 \mathrm{H}, \mathrm{CH}_{3}\right) ; 2.48-2.50\left(\mathrm{~d}, 2 \mathrm{H}, \mathrm{CH}_{2}\right)(\mathrm{J}=8 \mathrm{~Hz}) ; 3.1(\mathrm{~s}, 1 \mathrm{H}, \mathrm{CH}) ; 3.85(\mathrm{~s}, 2 \mathrm{H}$, $\left.\mathrm{NH}_{2}\right) ; 5.56(\mathrm{~s}, 1 \mathrm{H}, \mathrm{CH}) ; 7.1-7.6(\mathrm{~m}, 10 \mathrm{H}, \mathrm{Ar}-\mathrm{H}) ; 8.5\left(\mathrm{~d}, 2 \mathrm{H}, \mathrm{NH}_{2}\right) \mathrm{MS}(70 \mathrm{eV}): \mathrm{m} / \mathrm{z}=411.93[\mathrm{M}+]=100 \%$., found: 411.13; Anal. Calcd. for, $\mathrm{C}_{21} \mathrm{H}_{20} \mathrm{ClN}_{4} \mathrm{O}_{5} \mathrm{~S}$; 411.93: C, 61.23; H, 4.89; $\mathrm{Cl}, 8.61 ; \mathrm{N}, 13.60 ; \mathrm{O}, 3.88$; S,7.78. Found: C, 60.70; H, 4.22; N, 13.45; O, 13.58 S, 7.39. . ${ }^{13} \mathrm{C}$ NMR $\left(\mathrm{CDCl}_{3}\right): \delta=15.4,36.6,42.3$, $44.5,55.8,115.8,123.5,126.0,126.1,128.6,128.8,132.3,139.5,140.0,150.2,157.9,159.3$.

Synthesis of 5-(2-amino-6-(4 -chloro-3-methylphenyl)-5,6-dihydro-4H-1,3-thiazine-4-yl)-6-methyl-4(P-tolyl)-3,4-dihydropyrimidine-2(1H)-one(2f)

Crayola yellow Yield75\% mp $=207^{\circ} \mathrm{C}\left[\mathrm{C}_{21} \mathrm{H}_{20} \mathrm{ClN}_{4} \mathrm{O}_{5} \mathrm{~S}\right] \mathrm{IR}\left(\mathrm{KBr}, \lambda \max / \mathrm{cm}^{-1}\right) 3225,1682 \mathrm{~cm}^{-1}{ }^{1} \mathrm{HNMR}$ $\left(400 \mathrm{MHz}, \mathrm{CDCl}_{3}\right) \delta(\mathrm{ppm})=\delta 2.28\left(\mathrm{~s}, 3 \mathrm{H}, \mathrm{CH}_{3}\right) ; 2.48-2.50\left(\mathrm{~d}, 2 \mathrm{H}, \mathrm{CH}_{2}\right)(\mathrm{J}=8 \mathrm{~Hz}) ; 3.1(\mathrm{~s}, 1 \mathrm{H}, \mathrm{CH}) ; 3.85(\mathrm{~s}, 2 \mathrm{H}$, $\left.\mathrm{NH}_{2}\right) ; 5.56(\mathrm{~s}, 1 \mathrm{H}, \mathrm{CH}) ; 7.2-7.4(\mathrm{~m}, 9 \mathrm{H}, \mathrm{Ar}-\mathrm{H}) ; 8.5\left(\mathrm{~d}, 2 \mathrm{H}, \mathrm{NH}_{2}\right) \mathrm{MS}(70 \mathrm{eV}): \mathrm{m} / z=411.93[\mathrm{M}+]=100 \%$., found: 411.13; Anal. Calcd. for, $\mathrm{C}_{21} \mathrm{H}_{20} \mathrm{ClN}_{4} \mathrm{O}_{5} \mathrm{~S} ; 411.93$ : C, 61.23; $\mathrm{H}, 4.89 ; \mathrm{Cl}, 8.61 ; \mathrm{N}, 13.60 ; \mathrm{O}, 3.88$; S,7.78. Found: C, 60.70; H, 4.22; N, 12.45; O36.49.S, 6.39. ${ }^{13} \mathrm{C} \mathrm{NMR}\left(\mathrm{CDCl}_{3}\right): \delta=15.4,36.6,42.3$, $44.5,55.8,115.8,123.5,126.7,126.8,126.9,129.5,131.6,137.6,141.9,150.2,159.3$.

Synthesis of 5-(2-amino-6-(3,4 -dimethylphenyl)-5,6-dihydro-4H-1,3-thiazine-4-yl)-6-methyl-4-(4nitrophenyl)3,4-dihydropyrimidine-2(1H)-one $(2 \mathrm{~g})$

Peach yellow Yield $62 \% \mathrm{mp}=212^{0} \mathrm{C}\left[\mathrm{C}_{21} \mathrm{H}_{20} \mathrm{~N}_{5} \mathrm{O}_{3} \mathrm{~S}\right] \mathrm{IR}\left(\mathrm{KBr}, \lambda \max / \mathrm{cm}^{-1}\right) 3214,1672, \mathrm{~cm}^{-1}{ }^{1} \mathrm{HNMR}$ $\left(400 \mathrm{MHz}, \mathrm{CDCl}_{3}\right) \delta(\mathrm{ppm})=\delta 2.28\left(\mathrm{~s}, 3 \mathrm{H}, \mathrm{CH}_{3}\right) ; 2.48-2.50\left(\mathrm{~d}, 2 \mathrm{H}, \mathrm{CH}_{2}\right)(\mathrm{J}=8 \mathrm{~Hz}) ; 3.1(\mathrm{~s}, 1 \mathrm{H}, \mathrm{CH}) ; 3.85(\mathrm{~s}, 2 \mathrm{H}$, $\left.\mathrm{NH}_{2}\right) ; 5.56(\mathrm{~s}, 1 \mathrm{H}, \mathrm{CH}) ; 7.2-7.9(\mathrm{~m}, 7 \mathrm{H}, \mathrm{Ar}-\mathrm{H}) ; 8.1\left(\mathrm{~d}, 2 \mathrm{H}, \mathrm{CH}_{2}\right) ; 8.5\left(\mathrm{~d}, 2 \mathrm{H}, \mathrm{NH}_{2}\right) ; \mathrm{MS}(70 \mathrm{eV}): \mathrm{m} / z=$ 422.13[M+]=100\%., found: 422.13; Anal. Calcd. for, $\mathrm{C}_{21} \mathrm{H}_{20} \mathrm{~N}_{5} \mathrm{O}_{3} \mathrm{~S} ; 421.93$ : C, 59.70; H, 4.77; N, 16.58; O,11.36; S,7.50. Found: C, 58.11; H, 4.22; N, 16.45; O, 10.36. S, $6.39{ }^{13} \mathrm{C} \mathrm{NMR}\left(\mathrm{CDCl}_{3}\right): \delta=15.4,36.6$, $42.3,44.5,55.8,115.8,123.5,125.1,126.0,128.1,128.2,128.3,139.5,145.9,148.0,150.2,159.3$.

Synthesis of 5-(2-amino-6-(3-methyl-4-nitrophenyl)-5,6-dihydro-4H-1,3-thiazine-4-yl)-4-(4methoxyphenyl-)6-methyl-3,4-dihydropyrimidine-2(1H)-one (2h)

Amber yellow Yield $76 \% \mathrm{mp}=228^{0} \mathrm{C}\left[\mathrm{C}_{22} \mathrm{H}_{22} \mathrm{~N}_{5} \mathrm{O}_{4} \mathrm{~S}\right] \mathrm{IR}\left(\mathrm{KBr}, \lambda \max / \mathrm{cm}^{-1}\right) 3220,1674 \mathrm{~cm}^{-1}{ }^{1} \mathrm{HNMR}$ $\left(400 \mathrm{MHz}, \mathrm{CDCl}_{3}\right) \delta(\mathrm{ppm})=\delta 2.28\left(\mathrm{~s}, 3 \mathrm{H}, \mathrm{CH}_{3}\right) ; 2.48-2.50\left(\mathrm{~d}, 2 \mathrm{H}, \mathrm{CH}_{2}\right)(\mathrm{J}=8 \mathrm{~Hz}) ; 3.1(\mathrm{~s}, 1 \mathrm{H}, \mathrm{CH})$; 
RASĀYAN J. Chem.

Vol. 11 | No. 3 |1294 - 1299 | July - September | 2018

3.85(s,2H, $\left.\mathrm{NH}_{2}\right) ; 5.56(\mathrm{~s}, 1 \mathrm{H}, \mathrm{CH}) ; 7.2\left(\mathrm{~d}, 2 \mathrm{H}, \mathrm{CH}_{2}\right) ; 7.3-7.4(\mathrm{~m}, 4 \mathrm{H}, \mathrm{Ar}-\mathrm{H}) ; 8.2\left(\mathrm{~d}, 2 \mathrm{H}, \mathrm{CH}_{2}\right) ; 8.5(\mathrm{~d}, 2 \mathrm{H}$, $\mathrm{NH}_{2}$ ); MS $(70 \mathrm{eV}): \mathrm{m} / \mathrm{z}=452.14[\mathrm{M}+]=100 \%$., found: 452.14; Anal. Calcd. for, $\mathrm{C}_{22} \mathrm{H}_{22} \mathrm{~N}_{5} \mathrm{O}_{4} \mathrm{~S} ; 451.93: \mathrm{C}$, 58.39; H, 4.90; N, 15.48; O,14.14; S,7.09. Found: C, 57.11; H, 4.22; N, 14.45; O, 13.36. S, 6.39. ${ }^{13} \mathrm{C}$ NMR $\left(\mathrm{CDCl}_{3}\right): \delta=15.4,36.6,42.3,44.5,55.8,114.1,115.8,123.5,125.7,129.2,134.2,145.6,150.2$, 159.3 .

Synthesis 5-(2-amino-6-(4-chloro-3-methylphenyl))-5,6-dihydro-4H-1,3-thiazine-4-yl)-4-(4methoxyphenyl-)6-methyl-3,4-dihydropyrimidine-2(1H)-one(2i)

Canary yellow Yield $81 \% \mathrm{mp}=238^{0} \mathrm{C}\left[\mathrm{C}_{22} \mathrm{H}_{22} \mathrm{ClN}_{4} \mathrm{O}_{2} \mathrm{~S}\right] \mathrm{IR}\left(\mathrm{KBr}, \lambda \max / \mathrm{cm}^{-1}\right) 3227,1684 \mathrm{~cm}^{-1}{ }^{1} \mathrm{HNMR}$ $\left(400 \mathrm{MHz}, \mathrm{CDCl}_{3}\right) \delta(\mathrm{ppm})=\delta 2.28\left(\mathrm{~s}, 3 \mathrm{H}, \mathrm{CH}_{3}\right) ; 2.48-2.50\left(\mathrm{~d}, 2 \mathrm{H}, \mathrm{CH}_{2}\right)(\mathrm{J}=8 \mathrm{~Hz}) 3.1(\mathrm{~s}, 1 \mathrm{H}, \mathrm{CH}) ; 3.85(\mathrm{~s}, 2 \mathrm{H}$, $\left.\mathrm{NH}_{2}\right) ; 5.56(\mathrm{~s}, 1 \mathrm{H}, \mathrm{CH}) ; 6.8\left(\mathrm{~d}, 2 \mathrm{H}, \mathrm{CH}_{2}\right) ; 7.1-7.4(\mathrm{~m}, 6 \mathrm{H}, \mathrm{Ar}-\mathrm{H}) ; 8.5\left(\mathrm{~d}, 2 \mathrm{H}, \mathrm{NH}_{2}\right) \mathrm{MS}(70 \mathrm{eV}): \mathrm{m} / z=$ 441.12[M+]=100\%., found: 441.12; Anal. Calcd. for, $\mathrm{C}_{22} \mathrm{H}_{22} \mathrm{ClN}_{4} \mathrm{O}_{2} \mathrm{~S} ; 441.03$ : C, 59.79; H, 5.02; Cl, 8.02; N, 12.68; O,7.24; S,7.26. Found: C, 58.11; H, 4.22; Cl, 7.17; N, 12.45; O,6.67;.S,6.39. ${ }^{13} \mathrm{C}$ NMR $\left(\mathrm{CDCl}_{3}\right): \delta=15.4,36.6,42.3,44.5,55.8,58.8,114.1,115.8,123.5,125.7,128.8,128.9,131.6,134.2$, 137.6, 150.2, 158.6, 159.3.

Synthesis of 5-(2-amino-6-(4-methoxy- 3-methylphenyl))-5,6-dihydro-4H-1,3-thiazine-4-yl)-4-(4hydroxyphenyl-)6-methyl-3,4-dihydropyrimidine-2(1H)-one (2j)

Mustard yellow Yield $76 \% \mathrm{mp}=220^{\circ} \mathrm{C}\left[\mathrm{C}_{22} \mathrm{H}_{23} \mathrm{~N}_{4} \mathrm{O}_{3} \mathrm{~S}\right] \mathrm{IR}\left(\mathrm{KBr}, \lambda \mathrm{max} / \mathrm{cm}^{-1}\right) 3211,1674 \mathrm{~cm}^{-1}{ }^{1} \mathrm{HNMR}$ $\left(400 \mathrm{MHz}, \mathrm{CDCl}_{3}\right) \delta(\mathrm{ppm})=\delta 2.28\left(\mathrm{~s}, 3 \mathrm{H}, \mathrm{CH}_{3}\right) ; 2.48-2.50\left(\mathrm{~d}, 2 \mathrm{H}, \mathrm{CH}_{2}\right)(\mathrm{J}=8 \mathrm{~Hz}) 3.1(\mathrm{~s}, 1 \mathrm{H}, \mathrm{CH}) ; 3.85(\mathrm{~s}$, $\left.2 \mathrm{H}, \quad \mathrm{NH}_{2}\right) ; 5.56(\mathrm{~s}, 1 \mathrm{H}, \mathrm{CH}) ; 6.6-7.1(\mathrm{~m}, 8 \mathrm{H}, \mathrm{Ar}-\mathrm{H}) ; 8.5\left(\mathrm{~d}, 2 \mathrm{H}, \mathrm{NH}_{2}\right) ; \mathrm{MS}(70 \mathrm{eV}): \mathrm{m} / \mathrm{z}=$ 423.15[M+]=100\%., found: 422.10; Anal. Calcd. for, $\mathrm{C}_{22} \mathrm{H}_{23} \mathrm{~N}_{4} \mathrm{O}_{3} \mathrm{~S} ; 422.10$ : C, 62.39; H, 5.47; N, 13.23; $\mathrm{O}, 11.33$; S,7.57. Found: C, 61.11; H, 4.22; N, 12.45; O,11.30; S, 6.39. ${ }^{13} \mathrm{C} \mathrm{NMR}\left(\mathrm{CDCl}_{3}\right): \delta=15.4,36.6$, $42.3,44.5,55.8,58.8,114.4,115.8,123.5,126.1,129.1,131.8,150.2,156.5,157.9,159.3$.

\section{RESULTS AND DISCUSSION}

The synthesis of 5-cinnamoyl-6-methyl-4-phenyl-3,4-dihydropyrimidine2 $(1 \mathrm{H})$ - one using the catalytic amount of TBAB under dichloromethane and water as a biphasic solvent in 2:1 ratio has been accomplished. We optimized the reaction conditions with different phase transfer catalysts (Table 1)

Table-1: Different Phase Transfer Catalysts Used for the Synthesis of Thiazines

\begin{tabular}{c|c|c|c}
\hline S. No. & Catalyst & Time & Yield \\
\hline 1 & TBAB & $30 \mathrm{~min}$ & $84 \%$ \\
\hline 2 & PEG 400 & $60 \mathrm{~min}$ & $78 \%$ \\
\hline 3 & $\beta$ cyclodextrin & $90 \mathrm{~min}$ & $71 \%$ \\
\hline
\end{tabular}

The reaction of $\mathbf{1 a}$ in $\mathrm{CH}_{2} \mathrm{Cl}_{2}$-water with a catalytic amount of $\beta$-cyclodextrin at ambient temperature reaction was completed in 90 mins giving 2a in low yield. i.e 71\%. Table-1(Entry 3). The efficiency of the reaction was markedly influenced by the addition of PEG 400 in the biphasic system (Entry 2 ) at $50^{\circ} \mathrm{C}$ for 60 mins resulted in $78 \%$. However, the use of the solvent system of $\mathrm{CH}_{2} \mathrm{Cl}_{2}-\mathrm{H}_{2} \mathrm{O}$ under the influence of catalyst TBAB, resulted in dramatic enhancement of yield. (Entry 1) gives $84 \%$ yield within 30 mins. The use of TBAB resulted in higher reaction rates than those of reactions performed with other phase transfer catalysts. Remarkably it was observed that the reaction 1a proceed better in TBAB (Entry 1) compared with that performed in other catalysts (Entry 2,3). The reaction in TBAB unquestionably showed the highest level of efficacy and atom economy compared with other catalysts. The fine-tuning of the amount of catalyst was performed in the process. We initiated the reaction with $10 \mathrm{mmol} \%$ of catalyst and extensively experimented on the mole $\%$ of catalyst from $12.5 \mathrm{mmol} \%$ to $30 \mathrm{mmol} \%$.(Table- 2 ) . The performance of the catalyst could not be improved up to $20 \mathrm{mmol} \%$. The $30 \mathrm{mmol} \%$ of catalyst exhibited the superiority of reaction in a biphasic system with TBAB as a catalyst. The course of the reaction was monitored by thin layer chromatography.

Interestingly, $\mathrm{TBAB}$ in $\mathrm{CH}_{2} \mathrm{Cl}_{2}$-water solvent system proved to be exceptionally effective at enhancing the efficiency of the reaction. The critical role of TBAB in the reaction between two immiscible phases is to accelerate the reaction by making available the substrates at interfacial boundaries. The reactant $\mathbf{1 a}$ is 
RASĀYAN J. Chem.

Vol. 11 | No. 3 |1294 - 1299 | July - September | 2018

soluble in the aqueous phase at $50^{\circ} \mathrm{C}$ in presence of PTC when subjected to thermal agitation and is hydrophilic while another reactant i.e. thiourea is highly soluble in water.

Table-2: Fine Tuning of Catalyst for Synthesis of Substituted Thiazines

\begin{tabular}{c|c|c}
\hline S. No. & $\begin{array}{c}\text { \% of catalyst } \\
(\mathrm{mmol} \%)\end{array}$ & Yield \\
\hline 1 & 10 & No reaction \\
\hline 2 & 12.5 & $25 \%$ \\
\hline 3 & 16 & $27 \%$ \\
\hline 4 & 18 & $30 \%$ \\
\hline 5 & 20 & $32 \%$ \\
\hline 6 & 22 & $42 \%$ \\
\hline 7 & 25 & $68 \%$ \\
\hline 8 & 28 & $75 \%$ \\
\hline 9 & 30 & $84 \%$ \\
\hline
\end{tabular}

The different solvation affinities of the two reactants retard the reaction. The border between two mutually immiscible liquid phases is not a geometrical surface. Due to the interfacial mechanism, the crude product was obtained in the aqueous phase while and organic layer was separated by separating funnel. The filtrate was filtered to get the product and evaporated to remove water leaving behind the catalyst foe next cycle.

Table-3: Physical data of 5-(2-amino-6-(3,4-substituted phenyl)-5,6-dihydro-4H-1,3-thiazine-4-yl)-6-methyl-4(substituted tolyl)-3,4-dihydropyrimidine-2(1H)-one

\begin{tabular}{c|c|c|c|c|c}
\hline S. No. & $\mathrm{R}_{1}$ & $\mathrm{R}_{2}$ & $\mathrm{R}_{3}$ & $\begin{array}{c}\text { Melting } \\
\text { point }\left({ }^{\circ} \mathrm{C}\right)\end{array}$ & Yield(\%) \\
\hline $2 \mathrm{a}$ & $-\mathrm{H}$ & $-\mathrm{H}$ & $-\mathrm{H}$ & 192 & 84 \\
\hline $2 \mathrm{~b}$ & $-\mathrm{H}$ & $-\mathrm{NO}_{2}$ & $-\mathrm{H}$ & 210 & 75 \\
\hline $2 \mathrm{c}$ & $-\mathrm{H}$ & $-\mathrm{OCH}_{3}$ & $-\mathrm{H}$ & 205 & 79 \\
\hline $2 \mathrm{~d}$ & $-\mathrm{OCH}$ & $-\mathrm{OCH}_{3}$ & $-\mathrm{H}$ & 232 & 80 \\
\hline $2 \mathrm{e}$ & $-\mathrm{Cl}$ & $-\mathrm{H}$ & $-\mathrm{H}$ & 215 & 65 \\
\hline $2 \mathrm{f}$ & $-\mathrm{H}$ & $-\mathrm{Cl}$ & $-\mathrm{H}$ & 207 & 75 \\
\hline $2 \mathrm{~g}$ & $-\mathrm{NO}_{2}$ & $-\mathrm{H}$ & $-\mathrm{H}$ & 212 & 62 \\
\hline $2 \mathrm{~h}$ & $-\mathrm{OCH}_{3}$ & $-\mathrm{NO}_{2}$ & $-\mathrm{H}$ & 228 & 76 \\
\hline $2 \mathrm{i}$ & $-\mathrm{OCH}_{3}$ & $-\mathrm{Cl}_{3}$ & $-\mathrm{H}$ & 238 & 81 \\
\hline $2 \mathrm{j}$ & $-\mathrm{OH}$ & $-\mathrm{OCH}_{3}$ & $-\mathrm{H}$ & 220 & 76 \\
\hline
\end{tabular}

The IR spectra of compound 1a showed carbonyl absorption around 1663-1690 $\mathrm{cm}^{-1}$ and $-\mathrm{NH}$ absorption around $3230 \mathrm{~cm}^{-1}$. Moreover, the compound showed a mass ion peak of $100 \%$ intensity corresponding to their molecular weights in mass spectra further confirmed the structure. Furthermore, the ${ }^{1} \mathrm{HNMR}$ spectrum of compound 1a shows a singlet at $2.17 \mathrm{ppm}$ owing to three protons of $-\mathrm{CH}_{3}$ of the aromatic ring. A doublet at 6.56-6.59 ppm is assigned for one proton of -CO-CH group with $(\mathrm{J}=12.5 \mathrm{~Hz})$. The peak of the doublet at 6.52-6.55 ppm represents for one proton attached with an aromatic ring with $(\mathrm{J}=13.3 \mathrm{~Hz})$. A singlet for one proton of - $\mathrm{NH}$ appears at $4.92 \mathrm{ppm}$ and singlet of one proton of- $\mathrm{CH}$ corresponds at 5.09 ppm. A singlet for one proton of $-\mathrm{NH}$ appears at $5.26 \mathrm{ppm}$. The product $1 \mathbf{a}$ was analyzed for $\mathrm{C}_{20} \mathrm{H}_{1}{ }_{7} \mathrm{~N}_{2} \mathrm{O} 2$ which exhibited a molecular ion at $\mathrm{m} / \mathrm{z}=317.36[\mathrm{M}+1]$.

Contributing to the elucidation of the structure of compound 2a the infrared spectra showed the peaks at $3210,1670 \mathrm{~cm}^{-1}$. The $\mathrm{NH}$ absorption band corresponds at $3210 \mathrm{~cm}-{ }^{1}$ and a strong band at $1670 \mathrm{~cm}^{-1}$ shows the presence of carbonyl group $(\mathrm{C}=\mathrm{O})$. Furthermore, the ${ }^{1} \mathrm{HNMR}$ spectrum of compound 2a shows a singlet at $2.28 \mathrm{ppm}$ owing to three protons of $-\mathrm{CH}_{3}$ of Biginelli ring. A doublet at 2.4-2.5 ppm is 
assigned for two protons of $-\mathrm{CH}_{2}$ group with $(\mathrm{J}=10 \mathrm{~Hz})$. The peak of the triplet at $3.1 \mathrm{ppm}$ represents the$\mathrm{CH}$ of Biginelli ring. A singlet for two protons of $-\mathrm{NH}_{2}$ appears at $3.85 \mathrm{ppm}$ and singlet of one proton of$\mathrm{CH}$ corresponds at $5.57 \mathrm{ppm}$. The multiple at 7.1-7.6 ppm accorded for the aromatic protons. Two $-\mathrm{NH}$ singlets are observed at 8.1 and $8.5 \mathrm{ppm}$. In addition, ${ }^{1} \mathrm{HNMR}$ spectra of $\mathbf{2 c}$ and $2 \mathbf{d}$ shows the singlets for three protons of $-\mathrm{CH}_{3}$ at $3.83 \mathrm{ppm}$. The ${ }^{1} \mathrm{HNMR}$ spectra of $\mathbf{2} \mathbf{h}, \mathbf{2} \mathbf{j}$, and $\mathbf{2} \mathbf{i}$ show singlet for three protons of $-\mathrm{OCH}_{3}$ at $3.85 \mathrm{ppm} 3.87 \mathrm{ppm}$ and $3.89 \mathrm{ppm}$ respectively. The product $2 \mathrm{a}$ was analyzed for $\mathrm{C}_{9} \mathrm{H}_{7} \mathrm{ClN}_{2} \mathrm{OS}$ which exhibited a molecular ion at $\mathrm{m} / \mathrm{z}=377.14[\mathrm{M}+1]$.

\section{CONCLUSION}

In conclusion, DCM-water/TBAB proved to be an exceptionally efficient biphasic solvent system for the synthesis of thiazine derivatives at ambient temperature within $1 / 2$ hours. The protocol has the merit of being environmentally friendly and a simple operation, involving convenient workup, a short reaction time and resulting in good to excellent yields.

\section{ACKNOWLEDGMENT}

The authors express their gratitude to the Sophisticated Analytical Instrumentation Facility (SAIF), Chandigarh, for ${ }^{1} \mathrm{HNMR}$, FT-IR, Mass, CHN and ${ }^{13} \mathrm{CNMR}$ spectroscopic analysis.

\section{REFERENCES}

1. M. J.O. Donnell, Acc. Chem. Res., 37, 506(2004), DOI:10.1021/aro300625

2. (a) P.Balaswamy, S. Aravind, B. Satayanarayana, Rasayan J. Chem., 10(4), 1334(2017), DOI: 10.7324/RJC.2017.1041903 ; (b) H. Kuppuswamy, R.Venugopal, J .Chem and Chemical Sci.,2(1), 21(2012)

3. G. Deepika, P. Gopinath, G. Kranthi, C. Nagamani, C. Jayasree, Y.V.Naidu, N.V.S. Enaganti, J. Pharm. Res., 5, 1105(2012), DOI:10.3390/molecules 21081054

4. A.T. Chaviara, P.J. Cox, K. H. Repana, R. M. Papi, K.T. Papazisis, D. Zambouli, A.H. Kortsaris, D .A. Kyriakidis, C.A. Bolos, J. Inorg. Biochem., 98, 1271 (2004), DOI: 10.1080/14756360701841251

5. H. Joshi, P. Upadhyay, A. J Baxi, J. Indian Chem. Soc., 67, 779(1990)

6. (a) H. Kai, Y. Morioka, M. Tomida, T. Takahashi, M. Hattori, K. Hanasaki, K. Koike, H. Chiba, S. Shinohara, T. Kanemasa, Y. Iwamoto, K. Takahashi, Y. Yamaguchi, T. Baba, T. Yoshikawa, H. Takenaka, Bioorg. Med. Chem. Lett., 11, 3925( 2007), DOI: 10.3390/molecules 21081054; (b) H. Kai, Y. Morioka, T. Murashi, K. Morita, S. Shinonome, H. Nakazato K. Kawamoto, K. Hanasaki, F. Takahashi, S. Mihara, T. Arai, K. Abe, H. Okabe, T. Baba, T. Yoshikawa, H. Takenaka, Bioorg. Med. Chem. Lett., 11,4030( 2007), DOI:10:3109/14756360903389898

7. T. P. Trofimova, O. N. Zefirova, A. A. Mandrugin, V. M. Fedoseev, D. I. Peregud, M. V. Onufriev, N. V. Gulycaeva, S.Y. Proskuryakov, Chem. Bull., 63, 274(2008), DOI:10.3103/s0027131408050088

8. M. Koketsu, K. Tanaka, Y. Takenaka, C.D. Kwong, H. Ishihara, Eur. J. Pharm. Sci., 15, 307(2002), DOI: 10.1016/s0928-0987(02)000143

9. V.V Dabholkar, R.P. Gawande, Rasayan J. Chem., 3(4), 655(2010)

10. J.D. Bourzat, C. Cotrel, C. Guyon, Pitchen, Ph. US Patent 4994569, DOI: 10.1002/jhet.2690

11. J. Beauchamp, A.Benardeau, H. Hilpert, C.Migliorini, W. Riboulet, H. Wang, H. WO, Patent 2011/029803 A1, DOI:10.5958/0974-4150.2018.00026.3

[RJC-4012/2018] 